\title{
Wave Models for Offshore Wind Turbines
}

\author{
Puneet Agarwal ${ }^{\S}$ and Lance Manuel ${ }^{\mathrm{y}}$ \\ Department of Civil, Architectural, and Environmental Engineering \\ The University of Texas, Austin, TX 78712
}

\begin{abstract}
In the design of wind turbines - onshore or offshore - the prediction of extreme loads associated with a target (long) return period requires statistical extrapolation from available loads data. The data required for such extrapolation are obtained by stochastic timedomain simulation of the inflow turbulence and of the turbine response. Prediction of accurate loads depends on assumptions made in the simulation models employed, both for the turbine and for the input wind/wave conditions. While for the wind, inflow turbulence models are fairly well established due to the relative maturity of the industry for onshore wind energy development, for wave input, the current practice is to model irregular (random) waves using a linear wave theory. These wave modeling assumptions do not adequately represent waves in shallow waters where most offshore wind turbines are being sited. As an alternative to these less realistic (albeit simple) representations of ocean waves, the present study investigates the use of irregular nonlinear (second-order) waves for estimating loads on the support structure (monopile) of an offshore wind turbine. We use a 5MW utility-scale wind turbine model for the simulations. Using, first, the simpler linear irregular wave modeling assumptions, we establish long-term loads and identify controlling environmental conditions (i.e., the wind speed and wave height) that are associated with the 20-year return period load derived using the inverse first-order reliability method. We then present the theory for an improved nonlinear second-order irregular wave modeling approach which we use to compute hydrodynamic loads on a stand-alone monopile analyzed quasi-statically for the controlling long-term design environmental conditions identified from the linear model. We demonstrate that computed hydrodynamic loads are generally larger with the nonlinear wave modeling assumptions; this establishes the importance of using such refined nonlinear wave models in stochastic simulation of the response of offshore wind turbines. This motivates the need for continuing work directed towards incorporation of nonlinear irregular wave models in turbine response simulation studies and loads extrapolation for design.
\end{abstract}

\section{Introduction}

While addressing different load cases, wind turbine designers are required to estimate extreme and fatigue loads; this is usually done by carrying out stochastic turbine response time-domain simulations. The simulation of offshore wind turbine response involves simulations of the stochastic inflow wind field on the rotor plane and of the irregular (random) waves on the support structure. Once the wind and waves are simulated, the response of the turbine is computed in the time domain using an aeroelastic model of the turbine. Obtaining realistic response of the turbine depends, among other factors, on appropriate modeling of the incident wind and waves. The current practice for modeling waves on offshore wind turbines is limited to the representation of linear irregular waves. While such models are appropriate for deep waters, they are not accurate representations of waves in shallow waters where offshore wind turbines are most commonly sited. In shallow waters, waves are generally nonlinear in nature. It is, therefore, of interest to assess the influence of alternative wave models on the behavior of wind turbines (e.g., on the tower response) as well as on extrapolated long-term turbine loads. Nonlinear (second-order) irregular waves ${ }^{1-3}$ can better describe waves in shallow waters. In this study, we investigate differences in turbine response statistics and on long-term load predictions that arise from the use of alternative wave models.

\footnotetext{
${ }^{*}$ Graduate Research Assistant

${ }^{\dagger}$ Associate Professor
} 
The prediction of extreme loads for long return periods (on the order of 20-50 years, typically), as is required when addressing at least one of the design load cases specified in the International Electrotechnical Commission (IEC) guidelines, ${ }^{4}$ relies on extrapolation of load statistics from limited simulations. Extrapolation refers to estimation or prediction of a rare load fractile associated with the desired long return period. Several extrapolation techniques, such as direct integration of short-term distributions appropriately weighted by their likelihood of occurrence, as well as more efficient techniques such as the inverse first-order reliability method (inverse FORM) have been explored in wind turbine applications. ${ }^{5-7}$ While extrapolated load estimates are associated with a fair amount of statistical uncertainty, model uncertainty due to imperfect or unrealistic simulation models used may also result in errors in long-term load predictions. In this study, we will directly address the influence of model uncertainty as it pertains to the modeling of the waves. We will focus on how the sea surface elevation process, the water particle kinematics and, in particular, the hydrodynamic loads derived using a second-order nonlinear wave theory vary when nonlinear second-order waves are modeled as alternatives to the conventional linear first-order approach.

We use a utility-scale 5MW offshore wind turbine model developed at the National Renewable Energy Laboratory (NREL) $)^{8}$ in our simulation studies. The turbine is assumed to be sited in 20 meters of water. Stochastic time-domain simulations of the turbine response are performed using the computer program, FAST. ${ }^{9}$ We first discuss the short-term response of the wind turbine to linear irregular waves; this represents existing capability of offshore wind turbine response calculation in FAST. We focus only on tower loads (specifically, the fore-aft tower bending moment at the mudline here) since the influence of waves on loads on the rotor is not so significant, as has been demonstrated in other studies (see, for example, Agarwal and Manuel $\left.{ }^{10}\right)$. We briefly discuss the procedure for extrapolated long-term load prediction using the inverse FORM technique. We then present the theory related to the development of a second-order nonlinear irregular wave model for simulation and discuss how use of this model can result in different (usually larger) hydrodynamic loads on a cylinder equivalent to that of the support structure of our 5MW turbine. Finally, we discuss those circumstances where modeling nonlinear waves to derive realistic hydrodynamic loads may be most significant.

\section{Long-term Loads}

Design Load Case 1.1b of the IEC 61400-3 draft design guidelines ${ }^{4}$ recommends the use of statistical extrapolation to predict rare extreme turbine loads. Direct integration and the inverse FORM procedure are two common extrapolation methods. These are discussed briefly next.

\section{II.A. Direct Integration Method}

In direct integration, one estimates the turbine nominal load for design, $l_{T}$, associated with an acceptable probability of exceedance, $P_{T}$, or, equivalently, with a target return period of $T$ years, as follows:

$$
P_{T}=P\left[L>l_{T}\right]=\int_{\boldsymbol{X}} P\left[L>l_{T} \mid \boldsymbol{X}=\boldsymbol{x}\right] f_{\boldsymbol{X}}(\boldsymbol{x}) d \boldsymbol{x}
$$

where $f_{\boldsymbol{X}}(\boldsymbol{x})$ represents the joint probability density function of the environmental random variables, $\boldsymbol{X}$. For different trial values of the load, $l_{T}$, Eq. 1 enables one to compute the long-term probability by integrating the short-term load exceedance probability conditional on $\boldsymbol{X}$, i.e., $P\left[L>l_{T} \mid \boldsymbol{X}=\boldsymbol{x}\right]$, with the relative likelihood of different values of $\boldsymbol{X}$. This method, while exact, is expensive, as one is required to integrate over the entire domain of all the environmental random variables. In this study, two environmental random variables comprise $\boldsymbol{X}$ and are taken to be the ten-minute average wind speed, $V$, at hub height in the along-wind direction and the significant wave height, $H_{s}$, for waves assumed to be aligned with the waves.

\section{II.B. Inverse FORM}

Another extrapolation technique is the so-called inverse first-order reliability method (inverse FORM). ${ }^{11}$ Here, for the present application, one considers a surface in a three-dimensional space on one side of which (i.e., the "failure" side), it is assumed that $L>l_{T}$. The three dimensions of this space represent the jointly

distributed variables, $V, H_{s}$, and $L$, and it is possible to transform this space to an independent standard normal space $\boldsymbol{U}=\left(U_{1}, U_{2}, U_{3}\right)$, where the failure surface is a sphere of radius $\beta$ such that 


$$
u_{1}^{2}+u_{2}^{2}+u_{3}^{2}=\beta^{2}
$$

It is noted here that $\beta$ is directly related to the target probability of load exceedance; namely, $P_{T}=$ $\Phi(-\beta)$, where $\Phi()$ represents the cumulative distribution function of a standard normal random variable. The transformation of the random variables from the physical $\boldsymbol{X}$ space to the standard normal $\boldsymbol{U}$ space is carried out via the Rosenblatt transformation such that $F_{V}(v)=\Phi\left(u_{1}\right), F_{H \mid V}(h)=\Phi\left(u_{2}\right)$, and $F_{L \mid V, H}(l)=\Phi\left(u_{3}\right)$, where $F()$ denotes the cumulative distribution function in each case. The point on this failure surface where the load attains its maximum value is the "design" point, and this load represents the desired nominal $T$-year return period load, $l_{T}$. Possible differences between this long-term load estimate and one obtained using direct integration per Eq. 1 result only due to an assumed linearization of the limit state function in the inverse FORM approach; this linearization approximation is not very inaccurate for rare loads associated with very small target probabilities of exceedance. The reader is referred to other studies (e.g., Saranyasoontorn and $\mathrm{Manuel}^{7}$ ) for details on the inverse FORM approach applied to derive long-term wind turbine loads.

Both the extrapolation methods discussed above require data on load extremes, which must be obtained from turbine simulations. Any limitations or approximations inherent in a simulation model can influence the accuracy of long-term load predictions. One such model approximation in simulations is introduced in the conventional use of a linear theory to model the waves. In the following, we discuss the influence of nonlinear irregular waves on simulated turbine tower loads, and how it can alter extrapolated long-term loads.

\section{Turbine Response Simulation with Linear Irregular Waves}

\section{III.A. Simulation Model}

A $5 \mathrm{MW}$ wind turbine model developed at $\mathrm{NREL}^{8}$ closely representing utility-scale offshore wind turbines being manufactured today is used here. The turbine is a variable-speed, collective pitch-controlled machine with a maximum rotor speed of $12.1 \mathrm{rpm}$; its rated wind speed is $11.5 \mathrm{~m} / \mathrm{s}$. It is assumed to have a hub height that is 90 meters above the mean sea level, and a rotor diameter of 126 meters. It is assumed to be sited in 20 meters of water; it has a monopile support structure of $6 \mathrm{~m}$ diameter, which is assumed to be rigidly connected at the mudline. The turbine is assumed to be installed at an IEC Class I-B wind regime site. ${ }^{4,12}$ A Kaimal power spectrum and an exponential coherence spectrum are employed to describe the inflow turbulence random field over the rotor plane, which is simulated using the computer program, TurbSim. ${ }^{13}$

For the hydrodynamic loading on the support structure, irregular long-crested waves are simulated using a JONSWAP spectrum. ${ }^{14}$ This same wave spectrum is used for simulating linear and nonlinear irregular waves. Hydrodynamic loads are computed using Morison's equation; ${ }^{15,16}$ Wheeler stretching ${ }^{15}$ is used to account for the influence of the instantaneous sea surface elevation on water particle kinematics and hydrodynamic loads.

Once the time histories of the wind inflow turbulence field and the sea surface elevation are generated, stochastic time-domain simulations of the turbine response are performed using the computer program, FAST. FAST employs a combined modal and multi-body dynamics formulation for analysis of any turbine model. It models the tower and blades as flexible bodies and uses the first two bending modes in each of the longitudinal and transverse directions.

More details related to the modeling capabilities of

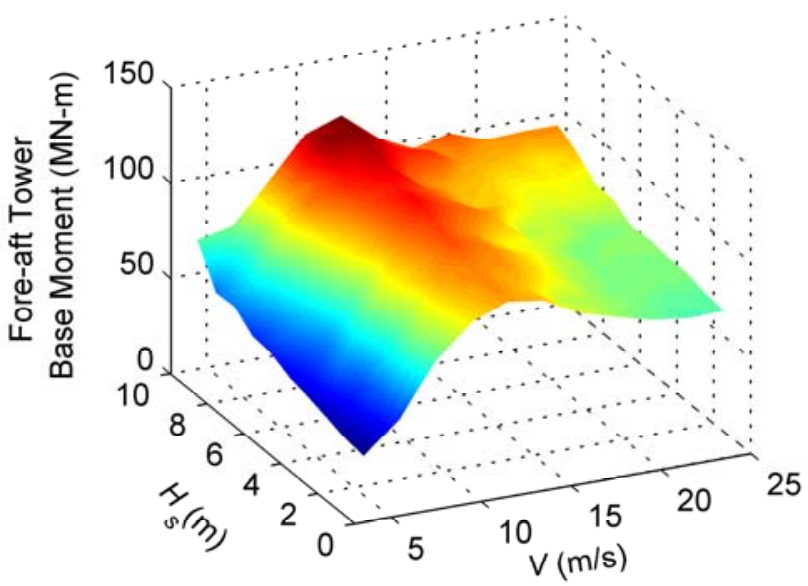

Figure 1. Variation with mean wind speed, $V$, and significant wave height, $H_{s}$, of the mean of the maximum values from six simulations of the fore-aft tower base moment. FAST may be found in the User's Guide. ${ }^{9}$ 


\section{III.B. Turbine Response}

In order to derive statistics or distributions of turbine loads conditional on wind speed and wave height, multiple simulations have to be carried out for selected pairs of mean wind speed and significant wave height. Figure 1 shows the average of ten-minute maximum fore-aft tower base moment from six simulations for a range of $\left(V, H_{s}\right)$ pairs considered. It is observed that this maximum load increases with wind speed, up to the rated wind speed of $11.5 \mathrm{~m} / \mathrm{s}$, and then decreases, as is expected due to blade-pitch control actions. Waves also have a clear influence on the turbine response; the maximum fore-aft tower base moment increases almost linearly with wave height. Important statistics for representative wind speeds and wave heights are summarized in Table 1. It can be seen that while the maximum load drops considerably as the wind speed is increased above rated, the standard deviation of the load does not. As wave heights are increased, both the maximum and standard deviation of the load increase. Hence, turbine long-term loads are expected to be governed by mean wind speeds near rated or by higher-than-rated wind speeds (where load variability may be larger) and by larger wave heights. More details related to specific turbine response statistics for different wind-wave combinations may be found in an earlier study by the authors. ${ }^{10}$

\begin{tabular}{|c|c|c|c|}
\hline \multirow{2}{*}{$\begin{array}{c}V \\
(\mathrm{~m} / \mathrm{s})\end{array}$} & \multirow{2}{*}{$\begin{array}{l}H_{s} \\
(\mathrm{~m})\end{array}$} & \multicolumn{2}{|c|}{ FATBM (MN-m) } \\
\hline & & Max & SD \\
\hline 4.0 & 4.5 & 39.4 & 8.6 \\
\hline 12.0 & 4.5 & 106.6 & 12.7 \\
\hline 24.0 & 4.5 & 78.4 & 12.3 \\
\hline 12.0 & 0.5 & 97.3 & 10.9 \\
\hline 12.0 & 4.5 & 106.6 & 12.7 \\
\hline 12.0 & 9.5 & 124.2 & 16.1 \\
\hline \multicolumn{4}{|c|}{$\begin{array}{l}V: \text { Hub-height } 10-\text { min average } \\
\text { wind speed, } H_{s}: \text { Significant wave } \\
\text { height, Max: Ten-minute maxi- } \\
\text { mum, SD: Standard deviation. }\end{array}$} \\
\hline
\end{tabular}

\section{III.C. Extrapolated Loads}

In a previous study, ${ }^{10}$ we discussed the use of inverse FORM for the same $5 \mathrm{MW}$ offshore turbine model as the one used here, and we showed that long-term loads derived using inverse FORM are as accurate as with the direct integration method. An additional advantage from using inverse FORM is that one can identify an environmental state (i.e., a $\left(V, H_{s}\right)$ pair) that governs the extrapolated long-term target load of interest. This desired long-term load is the load associated with a fractile, $p_{3}=\Phi\left(u_{3}\right)$, which can in turn be mapped to the short-term distribution of the load extremes, conditional on the environmental state. Specifically, based on Eq. 2, the fractile, $p_{3}$, corresponding to the target reliability index, $\beta$, is obtained as follows:

$$
p_{3}=\Phi\left(\sqrt{\beta^{2}-\left[\Phi^{-1}\left(F_{V}(v)\right)\right]^{2}-\left[\Phi^{-1}\left(F_{H \mid V}(h)\right)\right]^{2}}\right)
$$

For the IEC Class I-B wind regime site (for which our turbine model is being considered), we assume that the ten-minute average wind speed, $V$, at hub height has a mean value of $10 \mathrm{~m} / \mathrm{s}$ and that it can be described by a Rayleigh distribution. This distribution is truncated below the cut-in wind speed of $4 \mathrm{~m} / \mathrm{s}$ and above the cut-out wind speed of $24 \mathrm{~m} / \mathrm{s}$, since we are interested only in studying turbine loads during operation. The significant wave height, $H_{s}$, conditional on the mean wind speed, is assumed to be represented by a twoparameter Weibull distribution. The expected value of $H_{s}$ given $V$ is based upon the JONSWAP correlation 
between wind speed and wave height, ${ }^{15}$ while a coefficient of variation for $H_{s}$ given $V$ is assumed to be constant at 0.2 .

The load extremes required to establish the short-term distributions of load given wind speed and wave height are extracted from time series of the turbine load as global maxima, i.e., as the largest load experienced in each ten-minute simulation. We have shown ${ }^{10}$ that a mean wind speed of $16 \mathrm{~m} / \mathrm{s}$ and a significant wave height of $5.5 \mathrm{~m}$ is the governing environmental state that causes the tower base moment associated with a return period of 20 years. It was also shown that a large number of simulations are needed to obtain a stable short-term distribution for this environmental state and to estimate the $p_{3}$-quantile load, where $p_{3}$ is given by Eq. 3. An estimated distribution obtained from 150 ten-minute simulations is shown in Fig. 2. The exceedance probability, $\left(1-p_{3}\right)$, corresponding to the desired load fractile, $p_{3}$, computed from Eq. 3, is $3.87 \times 10^{-6}$ while the estimated maximum empirical exceedance fractile is several orders of magnitude higher at $6.60 \times 10^{-3}(=1 / 151)$. Clearly, extrapola-

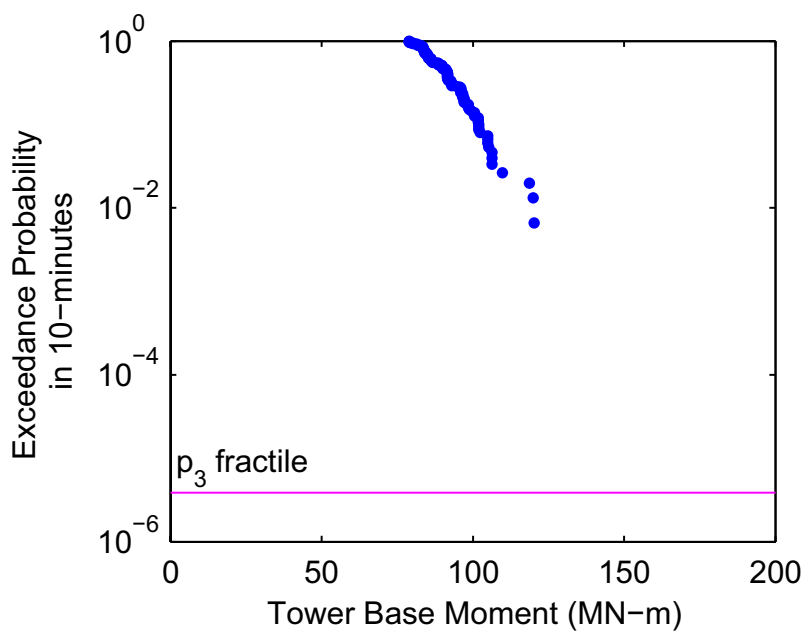

Figure 2. Empirical distribution of ten-minute maximum fore-aft tower base moment based on 150 simulations for a mean wind speed of $16 \mathrm{~m} / \mathrm{s}$ and a significant wave height of $5.5 \mathrm{~m}$. tion to the rarer probability is required. We obtain the desired 20-year load of $136.6 \mathrm{MN}-\mathrm{m}$ by fitting a two-parameter Weibull distribution to the tail of the empirical distribution data shown in Fig. 2. It was shown ${ }^{10}$ that this predicted based on inverse FORM is close to that predicted by direct integration.

\section{III.D. Limitations}

Long-term loads derived as presented above are based on simulation of irregular waves using a linear wave theory, which is not quite appropriate for waves in shallow waters, where waves have higher crests and shallower troughs than are predicted by the linear wave theory. This wave asymmetry results in a non-zero skewness of the stochastic sea surface elevation process; a linear irregular wave process is Gaussian with zero skewness. The use of a nonlinear wave theory makes it possible to model this wave asymmetry; this is required for more accurate wave kinematics too. Water particle velocities and accelerations predicted by a linear wave theory are generally smaller than those predicted by a nonlinear wave theory. Ultimately, hydrodynamic loads predicted by linear wave kinematics can also be unconservative. For these reasons, we seek to study here the influence of nonlinear waves on turbine tower loads.

\section{Second-order Nonlinear Irregular Waves}

Linear wave theory for regular or irregular waves involves solution of Laplace's equation expressed in terms of a velocity potential and the use of linearized boundary conditions. ${ }^{16}$ For nonlinear waves, modeling involves application of a perturbation approach to solve Laplace's equation with nonlinear boundary conditions. Longuet-Higgins ${ }^{17}$ used such an approach to derive a nonlinear wave theory for deep water. Hudspeth and $\mathrm{Chen}^{3}$ as well as Sharma and Dean ${ }^{2}$ used a similar approach to derive the theory for finite water depths. We will use the formulation of Sharma and Dean, which is briefly described below. This theory has increasingly been applied to a variety of problems in recent years. ${ }^{18-21}$

\section{IV.A. Theory}

The nonlinear sea surface elevation, $\eta(t)$, may be expressed as a sum of first- and second-order components:

$$
\eta(t)=\eta_{1}(t)+\eta_{2}(t)
$$

The first-order component, $\eta_{1}(t)$, is given as in linear wave theory by 


$$
\eta_{1}(t)=\sum_{k=1}^{N} A_{k} \cos \left(\omega_{k} t-\phi_{k}\right)
$$

where $\omega_{k}$ refers to the frequency of the $k^{t} h$ wave component and $\phi_{k}$ is the corresponding random phase assumed uniformly distributed over $[0,2 \pi]$. The amplitudes of the wave components, $A_{k}$, are Rayleigh distributed random variables whose mean square value is given as follows:

$$
E\left[A_{k}^{2}\right]=2 S\left(\omega_{k}\right) d \omega
$$

where $S\left(\omega_{k}\right)$ refers to the one-sided power spectral density function of the sea surface elevation process. The integer, $k$, in Eq. 5 refers to a frequency index that ranges from 1 to $N$, the total number of wave components represented in the simulated wave train.

The second-order component, $\eta_{2}(t)$, is obtained by the sum- and difference-frequency interactions as follows:

$$
\begin{gathered}
\eta_{2}(t)=\sum_{m=1}^{N} \sum_{n=1}^{N}\left[A_{m} A_{n}\left\{B_{m n}^{-} \cos \left(\psi_{m}-\psi_{n}\right)+B_{m n}^{+} \cos \left(\psi_{m}+\psi_{n}\right)\right\}\right] \\
\psi_{m}=\left(\omega_{m} t-\phi_{m}\right)
\end{gathered}
$$

The second-order transfer functions, $B_{m n}^{-}$and $B_{m n}^{+}$, are obtained from the solution of Laplace's equation for the velocity potential with nonlinear boundary conditions. They are functions of frequency and wave number, and are independent of the spectrum used. Equations for these transfer functions are given in the appendix. The equations for the velocity potential and those for the water particle velocity and acceleration are also given in the appendix. Second-order waves are thus obtained as a result of sum and difference interactions between pairs of frequencies. The phases of the second-order contributions are also determined by sum and difference interactions of the phases of the first-order component phases, which are random.

The simulation of irregular (random) linear or first-order waves, which involves a single summation (Eq. 5, can be efficiently performed using the Inverse Fast Fourier Transform (IFFT). Procedures for such linear wave simulation are well documented in the literature. ${ }^{22}$ Simulation of random nonlinear or second-order waves according to Eq. 7 involves a double summation, which can be more expensive. However, one can rewrite the double summation as a single summation by appropriately re-assembling and rewriting the coefficients of the double summation. Once all the coefficients for the equivalent single summation are assembled, one can use a one-dimensional IFFT as with linear waves to perform the simulations more efficiently.

\section{IV.B. Examples}

We now study two examples that illustrate the nature of the second-order nonlinearities and explain how it gives rise to asymmetry in the wave; these examples also serve as validations for the accuracy of the nonlinear irregular wave simulation procedure adopted.

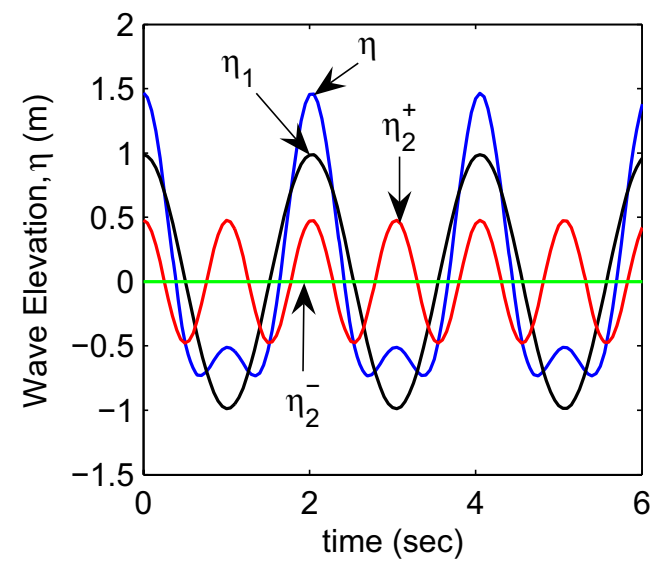

Figure 3. Simulation using a delta function power spectrum showing differences in linear and nonlinear waves.

\section{IV.B.1. Delta Function Spectrum}

Consider a delta function spectrum which is one with energy present at a single frequency, $\omega_{0}$ (taken to be 0.5 $\mathrm{Hz}$ here). Simulation of linear irregular wave for this spectrum leads, in effect, to a regular sinusoidal wave. Figure 3 shows that the sum-frequency component of the second order wave, $\eta_{2}^{+}$, is a wave at the frequency $2 \omega_{0}$. This component, when added to the first-order wave, results in systematically larger crests and shallower troughs; this is what gives rise to asymmetry in the waves and to a non-zero skewness of the sea surface 
elevation process. The difference-frequency component, $\eta_{2}^{-}$, corresponds to a zero-frequency signal which, in this case, has zero amplitude; for a spectrum with energy present at more than one frequency, the differencefrequency component results in a small set-down effect. For structures with low natural frequencies, the difference-frequency effect may become important.

\section{IV.B.2. JONSWAP Spectrum}

For real seas, the wave spectrum has energy over a range of frequencies, and a parametric spectral representation such as the JONSWAP spectrum is often used. The area under the power spectrum is equal to the variance of the simulated linear sea surface elevation process, a fact that can be used to check the accuracy of simulations. Likewise, the skewness of a simulated second-order sea surface elevation process should match a target skewness, which may be computed from knowledge of the spectrum and the second-order transfer functions, $B_{m n}^{-}$and $B_{m n}^{+}$. Langley ${ }^{23}$ developed a formulation to compute the target skewness, which we use here. This formulation can also aid in computing second and third moments of the first- and second-order sea surface elevation process.

We use a JONSWAP spectrum with a significant wave height of $12 \mathrm{~m}$ and peak spectral period of $14 \mathrm{sec}$ in this second example. The water depth is assumed to be $100 \mathrm{~m}$. This example was also used by Sweetman and Winterstein ${ }^{24}$ to demonstrate the capabilities of their computer program, WaveMaker, for simulating nonlinear waves (they did not, however, focus on kinematics and forces). Figure 4 shows a realization of the linear and nonlinear sea surface elevation processes. Clearly, larger crests are observed for the nonlinear waves, as one would expect. Table 2 shows that the variance, skewness and kurtosis of the sea surface elevation process, averaged over 1,000 simulations match well with target values computed from the spectrum. Further, the skewness value of 0.17 was also reported by Sweetman and Winterstein. ${ }^{24}$ This serves as a check of the accuracy of our simulations. An interesting aspect worth noting in Table 2 is the rather large coefficient of variation (COV) of $38 \%$ in the skewness value. Such large variation is expected as

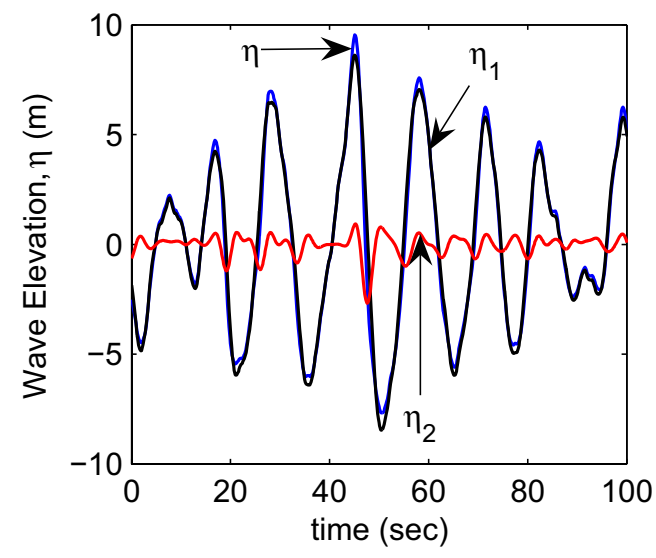

Figure 4. A time series simulation of linear and nonlinear waves based on a JONSWAP spectrum with $H_{s}=12 \mathrm{~m}$ and $T_{p}=14 \mathrm{sec}$. is discussed by $\mathrm{Hu}$ and Zhao. ${ }^{25}$ In fact, this is the reason why a large ensemble of random realizations is required if higher moments computed from time series are to match target values. Note that the non-zero skewness of steep waves, which is often observed in field measurements, cannot be captured by linear irregular wave theory and, thus, the use of nonlinear wave theory is desirable. ${ }^{19}$

Table 2. Statistical moments of the nonlinear sea surface elevation process simulated for a JONSWAP spectrum with $H_{s}=12 \mathrm{~m}$ and $T_{p}=14 \mathrm{sec}$.

\begin{tabular}{lccc}
\hline & Variance $\left(\mathrm{m}^{2}\right)$ & Skewness & Kurtosis \\
\hline Target $^{*}$ & 9.22 & 0.17 & 3.10 \\
Average* $^{* *}$ & 9.34 & 0.17 & 3.00 \\
COV $^{* *}$ & 0.15 & 0.38 & 0.10 \\
\hline${ }^{*}$ Computed from the spectrum (see Langley & \\
& & \\
${ }^{* *}$ Statistics based on 1,000 simulations.
\end{tabular}

\section{Loads on a Monopile due to Nonlinear Waves}

To understand the effect of wave loads, we study hydrodynamic loads on the monopile support structure of the turbine. The monopile is a cylinder of $6 \mathrm{~m}$ diameter, and the water depth is $20 \mathrm{~m}$. We compute water particle kinematics based on both linear and nonlinear irregular waves, while using the Wheeler stretching 
technique to compute kinematics up to the instantaneous sea surface. We use Morison's equation ${ }^{15}$ to compute hydrodynamic loads per unit length, $f$.

$$
f=f_{D}+f_{M}=\frac{1}{2} C_{D} \rho D u|u|+\frac{\pi D^{2}}{4} C_{M} \rho \dot{u}
$$

where $f_{D}$ and $f_{M}$ are drag and inertia forces, respectively, $C_{D}$ is the drag coefficient taken as 1.0, $C_{M}$ is the inertia coefficient taken as $2.0,{ }^{8} u$ is the horizontal water particle velocity, $\dot{u}$ is the horizontal water particle acceleration, $\rho$ is the density of water, and $D$ is the monopile diameter.

We assume that the monopile behaves quasi-statically, and we obtain the bending moment at the base based on a static analysis. Before going into detail regarding these loads, we discuss how closely these hydrodynamic loads on the stand-alone monopile represent the wave loads that result when the monopile is part of the turbine.

Table 3. Comparison of statistics of the tower base moment computed using FAST (with waves on the turbine, but without wind loads) and from a quasi-static analysis of the monopile.

\begin{tabular}{lccc}
\hline & $\begin{array}{c}\text { FAST } \\
\text { Linear Waves }\end{array}$ & $\begin{array}{c}\text { Quasi-static Monopile } \\
\text { Linear Waves }\end{array}$ & $\begin{array}{c}\text { Quasi-static Monopile } \\
\text { Nonlinear Waves }\end{array}$ \\
\hline Max (MN-m) & 25.96 & 27.00 & 33.92 \\
Std. Dev. (MN-m) & 7.91 & 7.65 & 8.01 \\
Skewness & -0.01 & 0.10 & 0.24 \\
Kurtosis & 3.21 & 3.29 & 4.07 \\
Peak Factor & 3.45 & 3.39 & 3.98 \\
\hline
\end{tabular}

\section{V.A. Monopile Loads with and without Turbine}

Table 3 shows statistics of the tower base moment from three types of analysis: (1) when the monopile is part of the turbine subjected to wave loads only (i.e., with no wind loads on the rotor) and the analysis is carried out using FAST which models linear waves only but considers flexibility and dynamics of the support structure; (2) when the monopile is assumed to be a stand-alone structure, without a turbine or any other associated mass, and a quasi-static analysis is carried out with linear waves; and (3) same as in 2 , but nonlinear irregular waves are used. The comparison of cases (1) and (2) should indicate how closely the stand-alone monopile (analyzed quasi-statically) represents the flexible monopile with the turbine. The comparison of cases (2) and (3), on the other hand, should indicate how much loads might differ when nonlinear waves are modeled instead of linear waves. Studying these three cases together might then help to anticipate the nature of loads in a fourth case - where the monopile as support structure to a wind turbine is subjected to nonlinear waves (this case is not studied here). The sea state for all three cases studied is the one that governed long-term loads for the turbine (discussed in Fig. 2) where linear waves were modeled, with a significant wave height of $5.5 \mathrm{~m}$ and spectral peak period of $11.2 \mathrm{sec}$. The results in Table 3 are averages over fifty simulations, except for the peak factors which are based on median extreme values of the load in ten minutes.

The maximum load on the monopile with turbine is about $26 \mathrm{MN}-\mathrm{m}$ (Table 3), which is very close to the load of $27 \mathrm{MN}-\mathrm{m}$ obtained for the stand-alone monopile analyzed quasi-statically with linear waves. The standard deviation and 10-min median extreme peak factor estimates for these two cases are also quite close, while higher moments (skewness and kurtosis) are more different. Figure 5(a) shows the power spectral density (PSD) function for the tower base moment when computed from FAST (with linear waves). The PSD shows two peaks; the first one at about $0.09 \mathrm{~Hz}$ corresponds to the spectral peak period of the waves which is $11.2 \mathrm{sec}$; the second peak at about $0.28 \mathrm{~Hz}$ corresponds to the natural period of the tower system. Clearly, these two peaks are spaced quite far apart; as such, linear waves are not expected to have a great influence on tower dynamics. The PSD of tower base moment computed for the stand-alone monopile with linear waves (Fig. 5(b)) also has a peak at the wave spectral peak period, but it has no other peak since the monopile was modeled quasi-statically. While the distribution of energy across different frequencies (i.e., the shape of the PSD) for these two cases is different, the total energy (which is the area under the spectrum, 


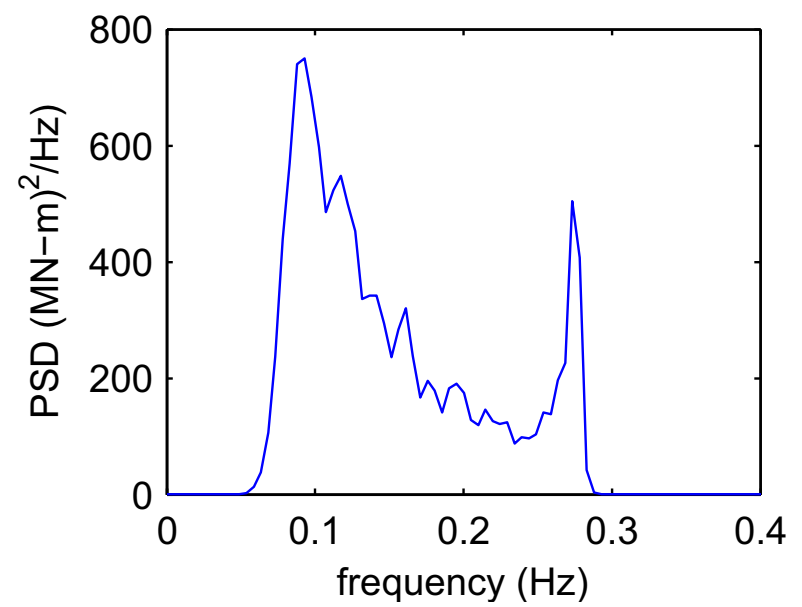

(a) Loads on monopile as obtained from FAST with loads only due to linear waves.

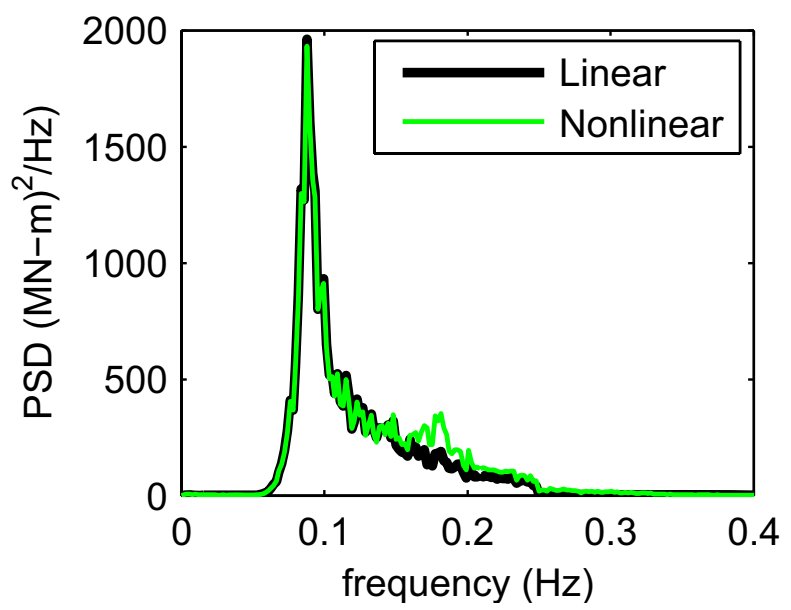

(b) Loads on a stand-alone monopile (without turbine) due to linear and nonlinear waves, analyzed quasi-statically.

Figure 5. Power spectra of tower base moment for $H_{s}=5.5 \mathrm{~m}$ and $T_{p}=11.2 \mathrm{sec}$.

also equal to variance of the process) is very close in the two cases, which is evident from the similar values of standard deviations (7.91 and 7.65 MN-m in Table 3). Based on these results, we can infer, as a first-order approximation that hydrodynamic loads obtained from a static analysis of a stand-alone monopile are a good representation of loads a monopile might experience when it is part of the turbine. The maximum load for the stand-alone monopile analyzed quasi-statically for nonlinear waves is $33.92 \mathrm{MN}-\mathrm{m}$, which is about $25 \%$ larger than that from linear waves. The power spectrum of the load for this case with nonlinear waves (Fig. 5(b)) shows an additional small peak at about $0.18 \mathrm{~Hz}$. Note that this peak occurs at about twice the spectral peak frequency of the waves; it arises due to the sum-frequency interaction that occurs in nonlinear waves. This additional peak might become important if the structure's natural period is close to it. In our case, however, the turbine tower's frequency $(0.28 \mathrm{~Hz})$ is much larger than $0.18 \mathrm{~Hz}$. Therefore, nonlinear waves are not expected to significantly contribute to tower dynamics.

It is also important to learn how important wave loads are, compared to wind loads, for the turbine. To understand this, we ran fifty simulations of turbine response with FAST when both wind and waves were included, when wind only was included, and when only waves were included (this last case was also one of the cases discussed above). The hub-height mean wind speed for these simulations was $16 \mathrm{~m} / \mathrm{s}$, and the significant wave height was $5.5 \mathrm{~m}$; again, this represents what was shown to be a governing environmental state for long-term loads. The random seeds for the simulations in each set of these three cases were kept same, so that a direct comparison could be made. The average of the maximum tower base moments was found to be $67.50,25.95$ and $88.31 \mathrm{MN}-\mathrm{m}$, respectively, for the wind-only, the wave-only, and the wind-andwave cases. Thus, wave-induced turbine loads are only about $30 \%$ of the total loads. Note that comparing maximum loads in this way is rather crude since both wind and wave loads are random processes and the maximum tower base moments due to both wind and waves do not simply add. Nevertheless, this quick calculation serves to give an idea of the relative importance of wind and wave loads. We next study in detail why loads due to linear and nonlinear irregular waves differ.

\section{V.B. Comparison of Loads due to Linear and Nonlinear Waves}

Table 3 shows that the maximum quasi-static load due to nonlinear waves on the monopile is $33.92 \mathrm{MN}-$ $\mathrm{m}$, which is about $25 \%$ larger than the corresponding maximum load due to linear waves; thus, nonlinear waves are clearly important. Moreover, the standard deviation, skewness, kurtosis, and peak factor are all larger with the nonlinear waves; differences in these statistics indicate a strongly non-Gaussian character introduced by the nonlinear waves to the load process. Large kurtosis values (greater than 3), for instance, imply fatter tails than for a Gaussian distribution; this implies that larger loads might be more likely to occur. The larger peak factors on ten-minute maximum loads seen with nonlinear waves are evidence of this and are indicative of maximum values systematically at higher levels above the mean value. What all of this suggests is that the ratio of extrapolated loads based on the use of nonlinear waves to that based on linear 
Table 4. Comparison of hydrodynamic loads on a stand-alone monopile (diameter $=6 \mathrm{~m}$, water depth $=20 \mathrm{~m}$ ) analyzed quasistatically and water particle kinematics, averaged over fifty simulations, computed with linear and nonlinear irregular waves using a JONSWAP spectrum with $H_{s}=5.5 \mathrm{~m}$ and $T_{p}=11.2 \mathrm{sec}$.

\begin{tabular}{lrrrrrr}
\hline & \multicolumn{3}{c}{ Tower Base Moment $(\mathrm{MN}-\mathrm{m})$} & & $\begin{array}{c}u_{\max } \\
(\mathrm{m} / \mathrm{s})\end{array}$ & $\begin{array}{c}\dot{u}_{\max } \\
\left(\mathrm{m} / \mathrm{s}^{2}\right)\end{array}$ \\
\cline { 2 - 4 } & Drag & Inertia & Total & & \\
\hline Linear & 7.88 & 25.68 & 27.00 & 3.69 & 2.84 \\
Nonlinear & 12.02 & 30.10 & 33.92 & & 4.55 & 3.42 \\
Ratio & 1.52 & 1.17 & 1.26 & 1.24 & 1.20 \\
\hline
\end{tabular}

Note: $u_{\max }$ and $\dot{u}_{\max }$ are maximum water particle velocity and acceleration, respectively, computed at the free surface.

waves could be larger than the corresponding ratio of $25 \%$ for maximum loads before extrapolation. Before investigating extreme loads in further detail, we discuss next the nature of hydrodynamic loads generated by modeling nonlinear waves.

The net hydrodynamic load experienced by the monopile is comprised of drag and inertia force contributions, which behave quite differently. We study their separate contributions to the maximum load (averaged over fifty simulations) in Table 4 . We also summarize estimates of the maximum horizontal particle velocity and acceleration at the free surface, $u_{\max }$ and $\dot{u}_{\max }$, respectively. It is clearly seen that maximum velocity and acceleration values increase by over $20 \%$ when nonlinear waves are modeled. When we look at the drag and inertia forces separately, we find that the drag forces increase by more than $50 \%$, while the inertia forces increase by only $17 \%$. This is mostly because drag forces are proportional to the square of velocity but also due possibly to a different wave profile and resulting higher crests and sometimes larger particle velocities found there in the simulations.

Another observation from Table 4 is that the total hydrodynamic loads on this monopile are dominated by inertia forces, both for linear as well as nonlinear waves. In the linear case, the ratio of the maximum tower base moment due to inertia forces to that due to drag forces is more than three; in the nonlinear case, this ratio about 2.5 (again, this is because of the greater influence of nonlinear waves on drag forces compared to inertia forces). For linear waves, whether a structure is drag- or inertia-dominated may be evaluated without carrying out time-domain simulations. To study the relative importance of drag versus inertia, one can examine the ratio of the standard deviations of the drag load to the inertia load (since both are random processes); this has been studied by others ${ }^{26,27}$ and the ratio can conveniently be written as follows:

$$
\begin{aligned}
\frac{\sigma\left[f_{D}\right]}{\sigma\left[f_{M}\right]} & =\sqrt{\frac{3}{4}} \kappa \\
\kappa & \approx \frac{C_{D}}{\pi C_{M}} \frac{H_{s}}{D} \frac{\cosh k(z+h)}{\sinh (k h)}
\end{aligned}
$$

where $\kappa$ is analogous to the Keulegan-Carpenter number for regular linear waves; also, $h$ is the water depth and $z$ refers to a vertical coordinate measured positive upwards and with origin at the mean sea level. Once a wave spectrum is given, one can evaluate Eq. 10 for a wave number corresponding, say, to the spectral peak period where wave energy is high. Since drag forces are maximum at crests, one can then obtain the maximum value of the ratio of the drag to inertia forces by assuming $z=H_{\max } / 2$, where $H_{\max }$ is the maximum instantaneous wave height which can be based on estimates of extremes of a Gaussian process representing the sea surface elevation. A very conservative estimate ${ }^{26}$ of $H_{\max }$ is $2 H_{s}$ which we use here. With these considerations and by using dimensions and other parameters specified for the monopile, the ratio in Eq. 10 is found to be approximately 0.2, which confirms that the monopile under consideration is dominated by inertia forces.

In Table 3, we presented peak factors for the tower base moment for three different types of analysis. In Table 5, we present peak factors on the ten-minute maximum tower base moment separately for drag 
Table 5. Peak factors on the ten-minute maximum tower base moment (median values based on 50 simulations).

\begin{tabular}{lrrr}
\hline & Drag & Inertia & Total \\
\hline Linear & 6.90 & 3.31 & 3.39 \\
Nonlinear & 8.76 & 3.70 & 3.98 \\
\hline
\end{tabular}

and inertia loads only for the stand-alone monopile analyzed quasi-statically using both linear and nonlinear waves. Based on an estimate of the number of zero-crossings of the load process, one can compute a theoretical peak factor assuming the process were Gaussian. ${ }^{26}$ Peak factors estimated in this way for the tower base moment process for drag forces alone, for inertia forces alone, and for the total hydrodynamic forces are all close to 3.0. Computed peak factors on the ten-minute maximum load (median value based on 50 simulations), however, are not always close to 3.0, as can be seen in Table 5. Even with linear waves, the peak factor for loads based on drag forces shows significant deviation from the Gaussian estimate compared to the peak factor based on inertia forces. This is expected since the squared velocity term in Eq. 9 makes the drag forces non-Gaussian even if the sea surface elevation and the water particle velocity processes are Gaussian, which is the case for linear waves. Load extremes (for any rare fractile of interest) for a process with a large peak factor will be larger than extremes predicted based on assuming a Gaussian process. Upon comparing peak factors for the linear and nonlinear waves cases in Table 5 , it is seen that the peak factor on loads for the nonlinear waves case is always larger than that for linear waves; even if only inertia forces are considered alone (no velocity squaring effect as for drag forces), the tower base moment is more nonGaussian as evidenced by the peak factor of around 3.7) due to nonlinearities and asymmetry introduced by the second-order wave theory. Finally, even though the monopile under investigation is inertia-dominated which can be verified by noting that the peak factors based on the total hydrodynamic forces are closer to the values based on inertia forces alone, the peak factor due to nonlinear waves is about $17 \%$ larger than that due to linear waves. Therefore, extreme load predictions based on nonlinear waves are expected to be generally be larger than those based on linear waves.

It is interesting that for this structure dominated by inertia forces, the nonlinear waves resulted in an increase in tower base moment of about $26 \%$ (see Table 4). We found in this study that going from linear to nonlinear waves introduces greater increases in particle velocities (and even greater when these are squared) than in particle accelerations; hence, the influence of nonlinear waves might be even more pronounced for drag-dominated structures - for example, for more slender members in space frames, tripods, etc. used as wind turbine support structures instead of monopiles. One can estimate the relative contribution of drag and inertia forces using Eq. 10, for example, where it can be seen that the influence of drag forces would increase for members of smaller size, especially if they are subjected to larger waves possible in deeper waters.

\section{V.C. Extreme Loads}

Our interest is in predicting extreme loads associated with rare (low) probability levels. Earlier, we discussed the subject of extrapolation of wind turbine loads in some detail, and derived ultimate loads for our 5MW wind turbine model for return period of 20 years, when linear irregular waves were used. We are interested in performing similar calculations for turbine loads using nonlinear waves. Such capability, 
however, is not yet fully integrated in simulation capability for wind turbines. Lacking this capability, it is of interest to study probability distributions on extreme loads due to waves only. To do this and to still relate back to our earlier extrapolated turbine support structure loads for the linear waves case, we return to the three cases we presented earlier: (1) turbine response simulations with FAST using linear waves and without any wind loads; (2) load simulations on a stand-alone monopile analyzed quasi-statically for linear waves; and (3) load simulations on a stand-alone stand-alone monopile analyzed quasi-statically for nonlinear waves. From 50 ten-minute simulations, we extracted global maxima of loads, and obtained the empirical distributions for tower base moment presented in Fig. 6. It can be seen that distributions of the tower base moment for the FAST simulations and for the stand-alone monopile (quasi-static load) with linear waves are quite close except in the tails. This is in line with our earlier observation that the loads (due to waves) on the monopile that supports the wind turbine are reasonably well represented by quasi-static loads on a stand-alone monopile. On the other hand, for the stand-alone monopile, the distributions of quasi-static load maxima for nonlinear and linear waves are strikingly different. Any rare load predicted by the nonlinear waves using extrapolation would be significantly larger than one predicted for linear waves. This observation is also consistent with our earlier discussion (in Table 3) about larger peak factors, and skewness and kurtosis estimates for nonlinear waves, all of which bring about larger rare (low-probability) loads. From Fig. 6, we might note that the largest simulated tower base moment (associated with an exceedance probability in ten minutes of $1 / 51$ or 0.0196 ) with the linear waves is approximately $50.3 \mathrm{MN}-\mathrm{m}$, while that for nonlinear waves is $78.4 \mathrm{MN}-\mathrm{m}$, more than $50 \%$ higher. Recall that the extreme tower base moment for the $5 \mathrm{MW}$ turbine, for a return period of 20 years, was predicted using linear waves to be $136.6 \mathrm{MN}-\mathrm{m}$ (see Fig. 2). Based on work presented here, we expect that hydrodynamic loads and resulting tower loads due to nonlinear waves at such long return periods will likely be larger than those due to linear waves by more than $50 \%$. Accordingly, we feel that it is important to further investigate and perhaps incorporate nonlinear wave modeling capability in the analysis of offshore wind turbines.

\begin{tabular}{|c|c|c|c|c|}
\hline \multicolumn{3}{|c|}{ Site } & \multicolumn{2}{|c|}{ Load Ratio } \\
\hline$H_{s}(\mathrm{~m})$ & $h(\mathrm{~m})$ & $T_{p}(\mathrm{sec})$ & $D=6 \mathrm{~m}$ & $D=3 \mathrm{~m}$ \\
\hline 4 & 10 & 9.1 & 1.39 & 1.79 \\
\hline 4 & 20 & 8.0 & 1.02 & 1.02 \\
\hline 8 & 20 & 12.9 & 1.70 & 1.87 \\
\hline 8 & 30 & 11.8 & 1.15 & 1.23 \\
\hline 8 & 40 & 11.3 & 1.04 & 1.07 \\
\hline 12 & 30 & 12.7 & 1.60 & 1.57 \\
\hline 12 & 40 & 14.7 & 1.08 & 1.33 \\
\hline 16 & 40 & 14.7 & 1.51 & 1.52 \\
\hline
\end{tabular}

$H_{s}$ : Significant wave height, $h$ : water depth, $T_{p}$ : Spectral peak period, $D$ : Monopile diameter.

\section{V.D. Load Comparisons for Different Sites}

Thus far, we have studied in detail the response of a 6-meter monopile subjected to a single sea state. It is of interest to study the influence of wave modeling assumptions for other cases as well. A few parameters that are of interest to study are monopile diameter, water depth, and significant wave height. Since we are interested in loads on offshore wind turbines, we chose representative values of these parameters based on a quick survey of existing offshore wind farms from which it was found that monopile diameters of the order of 3 to 6 meters and water depths ranging from 5 to 30 meters were common. For each water depth, we determined the maximum possible significant wave height, $H_{s}$, before waves would break; only $H_{s}$ values smaller than this maximum level were considered. The associated spectral peak period, $T_{p}$, for the waves 
was chosen as follows. Nonlinear waves developed according to the theory presented are assumed valid up to a steepness parameter, $s=H_{s} / L_{z}$, of about $0.08 .{ }^{25}$ Here, $L_{z}$ is the wavelength corresponding to the zerocrossing period. As a severe case scenario, we chose $s=0.06$ and computed $L_{z}$ for each choice of $H_{s}$. We then computed the zero-crossing period using the linear dispersion relation and from it, we determined the spectral peak period using a relation between the two periods based on the JONSWAP spectrum. ${ }^{21}$ Table 6 shows the ratio of the maximum quasi-static base moment (averaged over 50 ten-minute simulations) for a stand-alone monopile analyzed quasi-statically using nonlinear waves to that using linear waves.

Table 6 shows that monopile loads due to nonlinear waves can, in some cases, be more than $80 \%$ larger than loads due to linear waves. Clearly, for such sites, linear waves would give unconservative estimates of loads. Several other observations can be made from this table. For any site, the increased level of load due to the use of nonlinear waves is larger for a smaller diameter monpile. This is because smaller members are relatively more drag-dominated, where nonlinear waves are also more important. For a given wave height, the influence of nonlinear waves decreases with an increase in water depth. Therefore, modeling of nonlinear waves is important mainly at shallow-water sites where most offshore wind farms are usually located.

\section{V.E. Ongoing Work with Nonlinear Wave Modeling for Turbine Response Simulations}

Based on simulation studies on a stand-alone monopile only, we have shown that including models for nonlinear irregular waves can cause hydrodynamic loads to be more than $25 \%$ larger than those obtained using linear irregular waves. We are currently working on incorporating the nonlinear irregular wave modeling capability in the turbine simulation code, FAST, so that coupled aerodynamic and hydrodynamic analysis of offshore wind turbine may be performed. It is important that the work presented here is extended to full turbine response simulations.

\section{Summary and Conclusions}

Our objective in this study was to investigate long-term loads for a utility-scale 5MW offshore wind turbine sited in 20 meters of water. Our focus was on the fore-aft tower base moment at the mudline. We studied the influence of modeling nonlinear (second-order) irregular waves on hydrodynamic loads for a monopile support structure (a cylinder of $6 \mathrm{~m}$ diameter) of the turbine when compared to the more conventional linear wave modeling approach.

We used the inverse first-order reliability method to derive the extreme tower base moment for a 20-year return period for the $5 \mathrm{MW}$ turbine. The governing environmental state (mean wind speed and significant wave height) important for this 20-year load was also derived. For this governing significant wave height (of $5.5 \mathrm{~m}$ ), we investigated hydrodynamic loads on a stand-alone monopile support structure analyzed quasistatically. We presented the theory for modeling second-order irregular waves, which is more appropriate than the use of linear waves for shallow water depths. We showed that hydrodynamic loads on the standalone monopile computed using nonlinear waves could be more than $25 \%$ larger compared to those obtained using linear waves. Based on simulations, we estimated empirical short-term distribution of the base moment due to linear and nonlinear irregular waves; this clearly showed that loads corresponding to rare fractile levels that would be predicted if nonlinear waves were considered would be significantly larger than those predicted with linear irregular waves. Monopile loads due to nonlinear and linear waves was computed for a range of representative sites; it was found that loads due to nonlinear waves could be significantly larger than those from linear waves especially for shallow water sites and for more slender drag-dominated support structures.

In summary, this study has shown that nonlinear waves can have an important influence on the hydrodynamic loads experienced by an offshore wind turbine support structure, particularly in shallow waters. This motivates our interest in incorporating such nonlinear wave modeling capability into existing software for offshore wind turbine response simulation.

\section{Appendix}

\section{VII.A. Equations for Simulation of Second-Order Irregular Waves}

The transfer functions $B_{m n}^{-}$and $B_{m n}^{+}$in Eq. 7 are given by: 


$$
\begin{aligned}
& B_{m n}^{-}=\frac{1}{4}\left[\frac{D_{m n}^{-}-\left(k_{m} k_{n}+R_{m} R_{n}\right)}{\sqrt{R_{m} R_{n}}}+\left(R_{m}+R_{n}\right)\right] \\
& B_{m n}^{+}=\frac{1}{4}\left[\frac{D_{m n}^{+}-\left(k_{m} k_{n}-R_{m} R_{n}\right)}{\sqrt{R_{m} R_{n}}}+\left(R_{m}+R_{n}\right)\right]
\end{aligned}
$$

where

$$
\begin{aligned}
D_{m n}^{-}= & \frac{\left(\sqrt{R_{m}}-\sqrt{R_{n}}\right)\left\{\sqrt{R_{n}}\left(k_{m}^{2}-R_{m}^{2}\right)-\sqrt{R_{m}}\left(k_{n}^{2}-R_{n}^{2}\right)\right\}}{\left(\sqrt{R_{m}}-\sqrt{R_{n}}\right)^{2}-k_{m n}^{-} \tanh \left(k_{m n}^{-} h\right)} \\
& +\frac{2\left(\sqrt{R_{m}}-\sqrt{R_{n}}\right)^{2}\left(k_{m} k_{n}+R_{m} R_{n}\right)}{\left(\sqrt{R_{m}}-\sqrt{R_{n}}\right)^{2}-k_{m}^{-} \tanh \left(k_{m n}^{-} h\right)} \\
D_{m n}^{+}= & \frac{\left(\sqrt{R_{m}}+\sqrt{R_{n}}\right)\left\{\sqrt{R_{n}}\left(k_{m}^{2}-R_{m}^{2}\right)+\sqrt{R_{m}}\left(k_{n}^{2}-R_{n}^{2}\right)\right\}}{\left(\sqrt{R_{m}}+\sqrt{R_{n}}\right)^{2}-k_{m n}^{+} \tanh \left(k_{m n}^{+} h\right)} \\
& +\frac{2\left(\sqrt{R_{m}}+\sqrt{R_{n}}\right)^{2}\left(k_{m} k_{n}-R_{m} R_{n}\right)}{\left(\sqrt{R_{m}}+\sqrt{R_{n}}\right)^{2}-k_{m n}^{+} \tanh \left(k_{m n}^{+} h\right)}
\end{aligned}
$$

In the above, $k$ is the wave number which is related the frequency, $\omega$, and the water depth, $h$, via the dispersion relation. Related parameters that are needed are given as follows:

$$
\begin{aligned}
\omega^{2} & =g k \tanh (k h) \\
R_{m} & =\frac{\omega_{m}^{2}}{g} \\
k_{m n}^{-} & =\left|k_{m}-k_{n}\right| \\
k_{m n}^{+} & =k_{m}+k_{n}
\end{aligned}
$$

where $g$ refers to acceleration due to gravity.

The velocity potential, $\Phi$, is given as follows:

$$
\Phi=\Phi_{1}+\Phi_{2}
$$

The first-order velocity potential is:

$$
\Phi_{1}=\sum_{i=1}^{N} b_{i} \frac{\cosh \left(k_{i}(h+z)\right)}{\cosh \left(k_{i} h\right)} \sin \left(\omega_{i} t-\phi_{i}\right)
$$

The second-order velocity potential, $\Phi_{2}=\Phi_{2}^{-}+\Phi_{2}^{+}$, may be written as follows:

$$
\begin{aligned}
\Phi_{2}^{-} & =\frac{1}{4} \sum_{m=1}^{N} \sum_{n=1}^{N}\left[b_{m} b_{n} \frac{\cosh \left(k_{m n}^{-}(h+z)\right)}{\cosh \left(k_{m n}^{-} h\right)} \frac{D_{m n}^{-}}{\left(\omega_{m}-\omega_{n}\right)} \sin \left(\psi_{m}-\psi_{n}\right)\right] \\
\Phi_{2}^{+} & =\frac{1}{4} \sum_{m=1}^{N} \sum_{n=1}^{N}\left[b_{m} b_{n} \frac{\cosh \left(k_{m n}^{+}(h+z)\right)}{\cosh \left(k_{m n}^{+} h\right)} \frac{D_{m n}^{+}}{\left(\omega_{m}+\omega_{n}\right)} \sin \left(\psi_{m}+\psi_{n}\right)\right]
\end{aligned}
$$

where

$$
b_{m}=\frac{A_{m} g}{\omega_{m}}
$$

The horizontal water particle velocity, $u(z, t)$, may be obtained from the spatial derivative of the velocity potential as follows:

$$
u(z, t)=\frac{\partial \Phi}{\partial x}
$$

\section{4 of 15}


and the horizontal water particle acceleration is obtained by taking the time derivative of the velocity

$$
\dot{u}(z, t)=\frac{\partial u(z, t)}{\partial t}
$$

\section{Acknowledgments}

The authors gratefully acknowledge assistance received with the 5MW offshore wind turbine baseline model used in the simulation studies from Dr. Jason Jonkman of the National Renewable Energy Laboratory. The authors also wish to acknowledge the financial support provided by the National Science Foundation by way of two grants - CAREER Award No. CMMI-0449128 and Award No. CMMI-0727989.

\section{References}

${ }^{1}$ Sharma, J. N., Development and Evaluation of a Procedure for Simulating a Random Directional Second-order Sea
Surface and Associated Wave Forces, Ph.D. thesis, University of Delaware, Delaware, 1979.
${ }^{2}$ Sharma, J. N. and Dean, R. G., "Contributions to Second Order Directional Sea Simulation and Wave Forces," 26th
International Conference on Offshore Mechanics and Arctic Engineering, OMAE2007, Paper no. 29634, San Diego, CA, 2007.
${ }^{3}$ Hudspeth, R. and Chen, M.-C., "Digital Simulation of Nonlinear Random Waves," Journal of Waterway, Port, Coastal
and Ocean Division, ASCE, Vol. 105, No. WW1, 1979, pp. 67-85.
${ }^{4}$ IEC-61400-3, Wind Turbines - Part 3: Design Requirements for Offshore Wind Turbines, International Electrotechnical
Commission, TC88 WG3 Committee Draft, 2005.
${ }^{5}$ Moriarty, P. J., Holley, W. E., and Butterfield, S. P., "Extrapolation of Extreme and Fatigue Loads using Probabilistic
Methods," Tech. Rep. NREL/TP-500-34421, National Renewable Energy Laboratory, Golden, CO, 2006.
${ }^{6}$ Ragan, P. and Manuel, L., "Statistical Extrapolation Methods for Estimating Wind Turbine Extreme Loads," ASME
Wind Energy Symposium, AIAA, Reno, NV, 2007.
${ }^{7}$ Saranyasoontorn, K. and Manuel, L., "Design Loads for Wind Turbines using the Environmental Contour Method,"
Journal of Solar Energy Engineering, Transactions of the ASME, Vol. 128, No. 4, 2006, pp. 554-561.
${ }^{8}$ Jonkman, J. M., Butterfield, S., Musial, W., and Scott, G., "Definition of a 5-MW Reference Wind Turbine for Offshore System Development," Tech. Rep. NREL/TP-500-38060, National Renewable Energy Laboratory, Golden, CO, 2007 (to be published).

${ }^{9}$ Jonkman, J. M. and Buhl Jr., M. L., "FAST User's Guide," Tech. Rep. NREL/EL-500-38230, National Renewable Energy Laboratory, Golden, CO, 2005.

${ }^{10}$ Agarwal, P. and Manuel, L., "Simulation of Offshore Wind Turbine Response for Extreme Limit States," 26th International Conference on Offshore Mechanics and Arctic Engineering, OMAE2007, Paper no. 29326, San Diego, CA, 2007.

${ }^{11}$ Winterstein, S. R., Ude, T. C., Cornell, C. A., Bjerager, P., and Haver, S., "Environmental Contours for Extreme Response: Inverse FORM with Omission Factors," Proceedings, ICOSSAR-93, Innsbruck, Austria, 1993.

${ }^{12}$ IEC-61400-1, Wind Turbines - Part 1: Design Requirements, International Electrotechnical Commission, Ed. $3,2005$.

${ }^{13}$ Jonkman, B. J. and Buhl Jr., M. L., "TurbSim User's Guide," Tech. Rep. NREL/TP-500-41136, National Renewable Energy Laboratory, Golden, CO, 2007.

${ }^{14}$ DNV-OS-J101, Design of Offshore Wind Turbine Structures, Offshore Standard, Det Norske Veritas, 2007.

${ }^{15}$ Barltrop, N. and Adams, A., Dynamics of Fixed Marine Structures, Butterworth-Heinemann, London, 1991.

${ }^{16}$ Sarpkaya, T. and Issacson, M., Mechanics of Wave Forces on offshore Structures, Van Nostrand Reinhold, New York, 1981.

${ }^{17}$ Longuet-Higgins, M., "The Effect of Non-linearities on Statistical Distributions in the Theory of Sea Waves," Journal of Fluid Mechanics, Vol. 17, No. 3, 1963, pp. 459-480.

${ }^{18}$ Forristall, G., "Nonlinear Wave Calculations in Engineering Applications," Journal of Offshore Mechanics and Arctic Engineering, Vol. 124, 2002, pp. 28-33.

${ }^{19}$ Jha, A., Nonlinear Stochastic Models for Ocean Wave Loads and Responses of Offshore Structures and Vessels, Ph.D. thesis, Stanford University, 1997.

${ }^{20}$ ITTC, "The Specialist Committee on Environmental Modeliing, Final Report and Recommendations to the 22nd ITTC," Tech. rep., International Towing Tank Conference, Seoul, 1999.

${ }^{21}$ DNV-RP-C205, Environmental Conditions and Environmental Loads, Recommended Practice, Det Norske Veritas, 2007.

${ }^{22}$ Shinozuka, M. and Deodatis, G., "Simulation of Stochastic Processes by Spectral Representation," Applied Mechanics Reviews, Vol. 44, No. 4, 1991, pp. 191-203.

${ }^{23}$ Langley, R., "A Statistical Analysis of Nonlinear Random Waves," Ocean Engineering, Vol. 14, No. 5, 1987 , pp. 389-407.

${ }^{24}$ Sweetman, B. and Winterstein, S., "Second-order random Ocean Waves: Prediction of Temporal and Spatial Variation from Fixed and Moving References, The Routine WaveMaker," Tech. Rep. RMS-37, Civil Engineering Department, Stanford University, May 1999.

${ }^{25} \mathrm{Hu}$, S.-L. J. and Zhao, D., "Non-Gaussian Properties of Second-Order Random Waves," Journal of Engineering Mechanics, Vol. 119, No. 2, 1993, pp. 344-364.

${ }^{26}$ Madsen, H. O., Krenk, S., and Lind, N. C., Methods of Structural Safety, Prantice Hall, Englewood Cliffs, NJ, 1986.

${ }^{27}$ Ditlevsen, O., Stochastic Wave Loads for Tubular Offshore Structures, Models for Dynamics and Reliability Analysis, Section of Maritime Engineering, Department of Mechanical Engineering, Technical University of Denmark, 2003. 